\title{
Voltage-Gated Lipid Ion Channels
}

\author{
Andreas Blicher, Thomas Heimburg*
}

Membrane Biophysics Group, Niels Bohr Institute, University of Copenhagen, Copenhagen, Denmark

\begin{abstract}
Synthetic lipid membranes can display channel-like ion conduction events even in the absence of proteins. We show here that these events are voltage-gated with a quadratic voltage dependence as expected from electrostatic theory of capacitors. To this end, we recorded channel traces and current histograms in patch-experiments on lipid membranes. We derived a theoretical current-voltage relationship for pores in lipid membranes that describes the experimental data very well when assuming an asymmetric membrane. We determined the equilibrium constant between closed and open state and the open probability as a function of voltage. The voltage-dependence of the lipid pores is found comparable to that of protein channels. Lifetime distributions of open and closed events indicate that the channel open distribution does not follow exponential statistics but rather power law behavior for long open times.
\end{abstract}

Citation: Blicher A, Heimburg T (2013) Voltage-Gated Lipid lon Channels. PLoS ONE 8(6): e65707. doi:10.1371/journal.pone.0065707

Editor: Zoya Leonenko, University of Waterloo, Canada

Received November 19, 2012; Accepted April 29, 2013; Published June 18, 2013

Copyright: (c) 2013 Blicher, Heimburg. This is an open-access article distributed under the terms of the Creative Commons Attribution License, which permits unrestricted use, distribution, and reproduction in any medium, provided the original author and source are credited.

Funding: A.B. was supported by a grant from the University of Copenhagen. T.H. was supported by the Villum foundation (VKR 022130) - URL:http:// veluxfoundations.dk. The funders had no role in study design, data collection and analysis, decision to publish, or preparation of the manuscript.

Competing Interests: The authors have declared that no competing interests exist.

*E-mail: theimbu@nbi.dk

\section{Introduction}

Synthetic lipid bilayers can display channel-like conduction events through pores in the bilayer [1]. This finding was first reported by Yafuso and collaborators [2] on oxidized cholesterol membranes. Numerous subsequent reports show that one finds channel-like events in synthetic lipid membranes in the complete absence of proteins [3-10]. The channels reported in those studies are typically of similar conductance (several 10 to $100 \mathrm{pS}$ ) and lifetime (a few ms to several $100 \mathrm{~ms}$ ) than those of protein channels. In various studies in the recent decade, Colombini and collaborators reported that ceramide lipids may form pores in membranes with channel-like conduction appearance [11-16]. The authors propose that ceramide channels are stable structures (comparable to a porin, see tentative structure in [17]). The conductances of such pores are much larger (several nS), the lifetimes are longer (several seconds to minutes) and it is therefore uncertain whether ceramide-channels possess the same characteristics as other lipid pores, which rather resemble fluctuations in membrane density with amplitudes and lifetimes given by the thermal fluctuations of the lipid matrix (see below).

In the recent years, our own group has focused on the thermodynamics of membranes and in particular on the aspects of thermal fluctuations. Close to melting transitions the heat capacity of membranes is proportional to the lateral compressibility $[18,19]$. This is so because the fluctuations in enthalpy and the fluctuations in area are proportional. Furthermore, the lifetime of the fluctuations is also proportional to the excess heat capacity $[20,21]$. In agreement with this, previous experimental results suggest that the open likelihood and the open lifetime of lipid channels are directly related to fluctuation amplitudes and lifetimes. It implies, that in melting transitions the likelihood of finding pores in the membranes is high, and the open lifetime is long [22-24]. The latter effect is known as 'critical slowing-down'. The channel-like conduction events in membranes close to transitions just reflect the thermal fluctuations in the membrane, and the lifetime of the lipid channels corresponds to the fluctuation or relaxation time-scale, respectively [21]. This is important because biological membranes under physiological conditions in fact exist in a state close to melting transitions [25-27], and lipidbased channels can be expected under physiological conditions.

Recently, we have shown that the channel events in artificial membranes are strikingly similar to protein conduction ([28], demonstrated in comparison to various TRP channels) such that they cannot be distinguished by just investigating the traces. This finding suggests the possibility that the origin of ion channel events may not always be correctly assigned in the literature. The main difference between membranes in the absence and presence of channel proteins is that the membranes containing proteins coherently show a well-defined value for the single-channel conductance, while the channel conductance in pure lipid membranes is subject to considerable variations between individual experiments. The reason for this is presently not clear, but may originate from sample preparation and the influence of the pipette walls on the phase behavior of membranes.

Membrane transitions can be influenced by changes in various thermodynamic variables, e.g., hydrostatic and lateral pressure, calcium concentration (chemical potential of $\mathrm{Ca}^{2+}$ ), $\mathrm{pH}$ (chemical potential of $\mathrm{H}^{+}$), but also by small drugs such as general or local anesthetics [1]. Consequently, lipid channels are sensitive to anesthetics [22]. They can be blocked by anesthetics (such as octanol). This implies that drugs can act on channel events in the absence of any specific macromolecular receptor molecule. Rather, their effect is of a purely thermodynamic nature known as 'freezing-point depression' [27].

It is a common observation in the lab that lipid ion channels can be induced by voltage. Typically, the membrane ruptures at high voltage. At voltages somewhat below the rupture voltage one can induce channel formation [28]. In order to systematically study this effect in a quantitative manner one needs synthetic membrane 
preparations that are stable over a significant period of time in a large range of voltages. Close to transitions, this happens only rarely. Using black lipid membranes, Wodzinska et al. [29] showed the voltage dependence of channel formation in a dioleyl phosphatidylcholine:dipalmitoyl phosphatidylcholine $=2: 1$ mol:mol mixture in a range from $-220 \mathrm{mV}$ to $+220 \mathrm{~V}$. More than 300 individual traces of 30 second length could be analyzed. In this experiment, a symmetric current-voltage profile and a linear single-channel conductance of $\gamma \sim 330 \mathrm{pS}$ was found. The current traces clearly indicated that the channel open likelihood is a function of voltage (Fig. 2 in [29]). While only very few open events were found at voltages below $40 \mathrm{mV}$, the frequency and duration of channel open events increased significantly at higher voltages until the channels are open most of the time. While this represents a proof-of-concept for the voltage-gating of lipid pores, the authors unfortunately did not analyze the voltage dependence of the open probability distribution in more detail.

In this publication, we demonstrate quantitatively that lipid channels may be gated by voltage. The effect of voltage on black lipid membranes was investigated by several authors in the 1970s. It was found that charging of the membrane capacitor generates a force on the membrane that leads to an effective reduction of the membrane thickness and thereby to an increase in capacitance with a quadratic voltage dependence. It has been proposed that electroporation (the generation of voltage-induce pores in bilayers) is a consequence of these forces [30-33]. Recently, we proposed that the electrostatic forces associated with an excess charge can also induce melting transitions in membranes [34]. Such transitions would influence the elastic properties of the membrane and thereby the likelihood of pore formation in the membrane [22]. Further, due to the coupling to fluctuation lifetimes, voltage can influence the open timescales of the lipid channels. In previous reports we have demonstrated that membranes can display nonlinear current-voltage relationships [28,29] and that lipid channels can be induced by voltage. Here, we study the voltage-gating of lipid channels more systematically and propose a theoretical description for inward or outward rectified I-V curves of synthetic lipid membranes that is in excellent agreement with experimental data.

\section{Materials and Methods}

Lipids (1,2-dimyristoyl-sn-glycero-3-phosphocholine - DMPC; 1,2-dilaureoyl-sn-glycero-3-phosphocholine - DLPG) were purchased from Avanti Polar Lipids (Alabaster, Al). In all experiments shown here, we used a DMPG:DLPC $=10: 1 \mathrm{~mol} / \mathrm{mol} \mathrm{mixture.}$ The $150 \mathrm{mM} \mathrm{NaCl}$ electrolyte solutions used for the patch clamp experiments were buffered with 2 mM HEPES (Sigma-Aldrich, Germany) and $1 \mathrm{mM}$ disodium EDTA (Fluka, Switzerland). The $\mathrm{pH}$ was adjusted to 7.4 using $\mathrm{NaOH}$. The specific conductivity of the solution was measured to be $1.412 \mathrm{~S} / \mathrm{m}$ at $21^{\circ} \mathrm{C}$. In order to get rid of dust particles and other possible contaminants, the buffer was always pressed through a sterile $0.2 \mu \mathrm{m}$ filter $\left(\right.$ Minisart ${ }^{\circledR}$ \#16532, Sartorius Stedim Biotech, France) immediately before use. All chemicals used were of the highest purity grade available and any water used was purified (resistivity $>18 \mathrm{M} \Omega \cdot \mathrm{cm}$ ) on a desktop EASYpure RF water purification system from Barnstead/ Thermolyne (Dubuque/IA, USA).

Each lipid type was dissolved separately in an organic solvent (dichloromethane : methanol=2:1). The two components were then mixed in the desired ratio. This mixture was dried by gentle heating while being exposed to an air stream. The remaining organic solvent was then removed by placing the sample in a high vacuum desiccator for a couple of hours. The resulting lipid film was then resuspended by adding a highly volatile solvent (hexane:ethanol 4:1 by volume). The final lipid concentration in the solvent was $\sim 1-2 \mathrm{mM}$.

Electrophysiological measurements were performed on single synthetic membranes spanned over the tip of a patch pipette using the tip-dipping method [35]. In this method, the planar lipid bilayer membrane is formed on the tip of a patch-clamp glass pipette that has been filled with electrolyte solution. The tip is in contact with the surface of a beaker filled with the same electrolyte solution. The lipid solution in the hexane/ethanol mixture is then brought into contact with the outer surface of the glass pipette. When the solution flows down the pipette, a membrane spontaneously forms at the tip of the pipette. The solvent was allowed to evaporate for at least 30 seconds before the pipette was lowered $45 \mathrm{~mm}$ below the bath surface. The main advantage of this method is that the resulting membrane is practically solvent free.

The patch diameter was $\sim 1 \mu \mathrm{m}$. Pipettes were pulled from $1.5 \mathrm{~mm} / 0.84 \mathrm{~mm}$ (outer diameter/inner diameter) borosilicate glass capillaries (\#1B150F-3, World Precision Instruments, USA) with a vertical PG-10 puller from Narishige Group, Japan. Current recordings were made using an Axopatch 200B patch clamp amplifier (Axon Instruments Inc., Union City/CA, USA). The pipette and electrodes were mounted on a cooled capacitor feed- back integrating headstage amplifier (Headstage CV 203BU, Axon Instruments Inc.). The same experimental procedure was used and described in [28].

Channel kinetics has been determined by defining a suitable threshold for an open event in an automated procedure. The threshold was set to be $\pm 4 \sigma$, where $\sigma$ is the noise of the baseline current. The method is described in more detail in the $\mathrm{PhD}$ thesis of one of the authors [36]. Somewhat more sophisticated methods to bin the kinetic data in a logarithmic manner can be found in [37-39].

The patch-pipette experiments have the advantage over black lipid membrane experiments that they result in much more stable and consistent conductance traces, and the membranes don't rupture so easily. However, due to the small patch size, there seems to be a large influence of the pipette on the membranes. Newly formed membranes over fresh pipettes lead to somewhat varying quantitative results. The voltage dependence of our membranes was therefore always recorded for one unique membrane and one pipette. While trends and qualitative behavior are reproducible between different experiments, the absolute numbers vary (see [28] for more details).

\section{Theory}

The electrostatic force, $\mathcal{F}$, exerted on a planar membrane by a voltage is given by

$$
\mathcal{F}=\frac{1}{2} \frac{C_{m} V_{m}^{2}}{D}
$$

where $C_{m}$ is the membrane capacitance, $V_{m}$ is the transmembrane voltage and $D$ is the membrane thickness [34]. This force reduces the thickness of the membrane [40]. The electrical work performed on the membrane by a change in thickness from $D_{1}$ to $D_{2}$ is

$$
\Delta W_{e l}=\int_{D_{1}}^{D_{2}} \mathcal{F} d D=\frac{1}{2} \epsilon_{0} V_{m}^{2} \int_{D_{1}}^{D_{2}} \epsilon \frac{A}{D^{2}} d D \equiv \alpha V_{m}^{2}
$$


where $A$ is area, $\epsilon_{0}$ is the vacuum permittivity, and $\varepsilon$ is the dielectric constant of the membrane core. The coefficient $\alpha$ is constant for constant temperature and pressure. This work is proportional to the square of the voltage, and one would thus reasonably assume that the free energy of pore formation is related to the square of the voltage and to the elastic constants of the membrane. This has been proposed previously $[32,41]$. Therefore, the free energy for pore formation has the form

$$
\Delta G=\Delta G_{0}+\alpha V_{m}^{2}
$$

where $\Delta G_{0}$ is the free energy difference between open and closed pore in the absence of voltage. $\Delta G_{0}$ reflects the elastic properties of the membrane that depend on composition, temperature and pressure. Such quadratic dependence is reasonable for symmetric membranes because a linear term can be excluded for symmetry reasons. For asymmetric membranes one obtains

$$
\Delta G=\Delta G_{0}+\alpha\left(V_{m}-V_{0}\right)^{2},
$$

where the offset voltage $V_{0}$ may be due to membrane curvature, the presence of the pipette wall on one side of the membrane or to a different lipid composition in the two membrane leaflets [42]. Membrane curvature could possibly originate from slight pressure difference on the two sides of the membrane due to suction or dipping the pipette tip deeper into the aqueous medium (see Materials section). At the end of the 'Results and Discussion' section we outline how such a curvature can create a transmembrane voltage on the order of $100 \mathrm{mV}$ associated to a phenomenon called flexoelectricity.

The probability, $P_{\text {open }}\left(V_{m}\right)$, of finding an open pore in the membrane at a fixed voltage is given by

$$
P_{\text {open }}\left(V_{m}\right)=\frac{K\left(V_{m}\right)}{1+K\left(V_{m}\right)} \quad ; \quad K\left(V_{m}\right)=\exp \left(-\frac{\Delta G}{k T}\right),
$$

where $K\left(V_{m}\right)$ is the voltage-dependent equilibrium constant between open and closed states of a single pore.

The current-voltage relation for the lipid membrane is proportional to the likelihood of finding an open channel for a given voltage:

$$
I_{m}=\gamma_{p} \cdot P_{\text {open }} \cdot\left(V_{m}-E_{0}\right)
$$

where $\gamma_{p}$ is the conductance of a single pore (or $\mathrm{N}$ identical pores) and $E_{0}=$ is the Nernst potential of a particular ion with charge $z$ given by $E_{0}=(R T / z F) \ln \left(\left[C_{\text {out }}\right] /\left[C_{\text {in }}\right]\right)$. While the voltage $V_{0}$ reflects the asymmetry of the membrane, $E_{0}$ reflects the asymmetry of the ion concentrations of the buffer solution. If the aqueous buffer is the same on both sides of the membrane, the Nernst potential is zero. Eqs. 4-6 contain the theoretical description for the $\mathrm{I}-\mathrm{V}$ curves of lipid channels. It is based on the concept of forces induced by charging the membrane capacitor.

The above simple capacitor model differs from the commonly used Eyring-type activation scheme of form

$I=z F k_{0}\left[[C]_{\text {in }} \exp \left(\frac{\delta z F V_{m}}{R T}\right)-[C]_{\text {out }} \exp \left(-\frac{(1-\delta) z F V_{m}}{R T}\right)\right]$

that assumes flow of charges in a field and a linear dependence of the free energy of the conduction rates on voltage. Here, $\delta$ is the position of an activation barrier in the membrane. The HodgkinHuxley gate model makes use of such an activation scheme [43].

\section{Results and Discussion}

We performed patch recordings on a DMPC : DLPC $=10: 1$ membrane [44] at $\mathrm{T}=30^{\circ} \mathrm{C}$ in a $150 \mathrm{mM} \mathrm{KCl}$ buffer. This mixture displays a chain melting transition close to room temperature [28]. Fig. 1 (left) shows a time sequence of the changes in total membrane current when voltage is changed stepwise. Around $50-60 \mathrm{mV}$ one finds small quantized steps on the current trace which are not always well resolved (insert to Fig. 1, left). The single channel conductance of these steps is $\approx 125 \mathrm{pS}$. This value is small compared to the overall conductance of the total membrane at this voltage of $\approx 4.7 \mathrm{nS}$. At other voltages, no single channels were observed. The asymmetry of the membrane becomes apparent in Fig. 1 (right), which shows the current-voltage relation for the total membrane current including baseline current. The solid line represents a fit to eq. 6 yielding $V_{0}=-110 \mathrm{mV}, \quad \gamma_{p}=6.62 \mathrm{nS}, \quad \Delta G_{0}=5.2 \mathrm{~kJ} / \mathrm{mol}, \quad$ and $\left.\alpha=-248 \mathrm{~kJ} / \mathrm{mol} \cdot \mathrm{V}^{2}\right)$. This fit reproduces the experimental current-voltage profile very well. Such an outward rectified I-V curve for a synthetic membrane was already shown in [28] where it was analyzed with an Eyring transition state model (eq. 7) that is very commonly used in the protein channel field. The present theoretical description yields a much superior description of the experimental data. Eq. 6 is based on the assumption of a single channel that opens in a voltage-dependent manner. The open likelihood of this channel (eq. 5) is shown in the insert to the right hand panel of Fig. 1. The present theoretical treatment also yields very good descriptions of the $\mathrm{I}-\mathrm{V}$ profiles of various transient receptor potential (TRP) channels reconstituted in human embryonic kidney cells with very similar parameters as used for the synthetic membrane (cf., [28], fits shown in [45]). The I-V curves of the TRP channels were also described with much less success by the Eyring model [28].

Two conclusions can be drawn from the I-V curve (Fig. 1):

1. There are net currents through the membrane even under conditions where individual channels cannot be visually resolved. These currents are well-described by a simple model that assumes the formation of pores with well-defined size. If such pores in fact exist, they must be short lived or much smaller than the relatively big conductance steps in the Figures discussed below.

2. In this particular experiment, the offset-voltage of the membrane in the patch pipette is $V_{0}=-110 \mathrm{mV}$, which indicates that the membrane is asymmetric. In the BLM experiment by Wodzinska et al. [29] discussed in the introduction, $V_{0}$ was $\approx 0 \mathrm{mV}$, indicating a symmetric membrane. It is a general observation in our lab that BLM measurements on large membranes tend to result in more symmetric current-voltage relations than patch recordings with small tips diameters.

Fig. 1 also demonstrates an experimental problem with obtaining statistically relevant data: The membrane has to be stable over the whole voltage regime of interest, and the overall properties have to remain constant during the total time of the experiment. In Fig. 1, the currents at voltages between $+70 \mathrm{mV}$ and $+90 \mathrm{mV}$ were recorded three times within two minutes. The variations during this time show up in the large noise in the respective interval in the $\mathrm{I}-\mathrm{V}$ curve. Obviously, the membrane properties drift slightly during the experiment. While the voltage- 

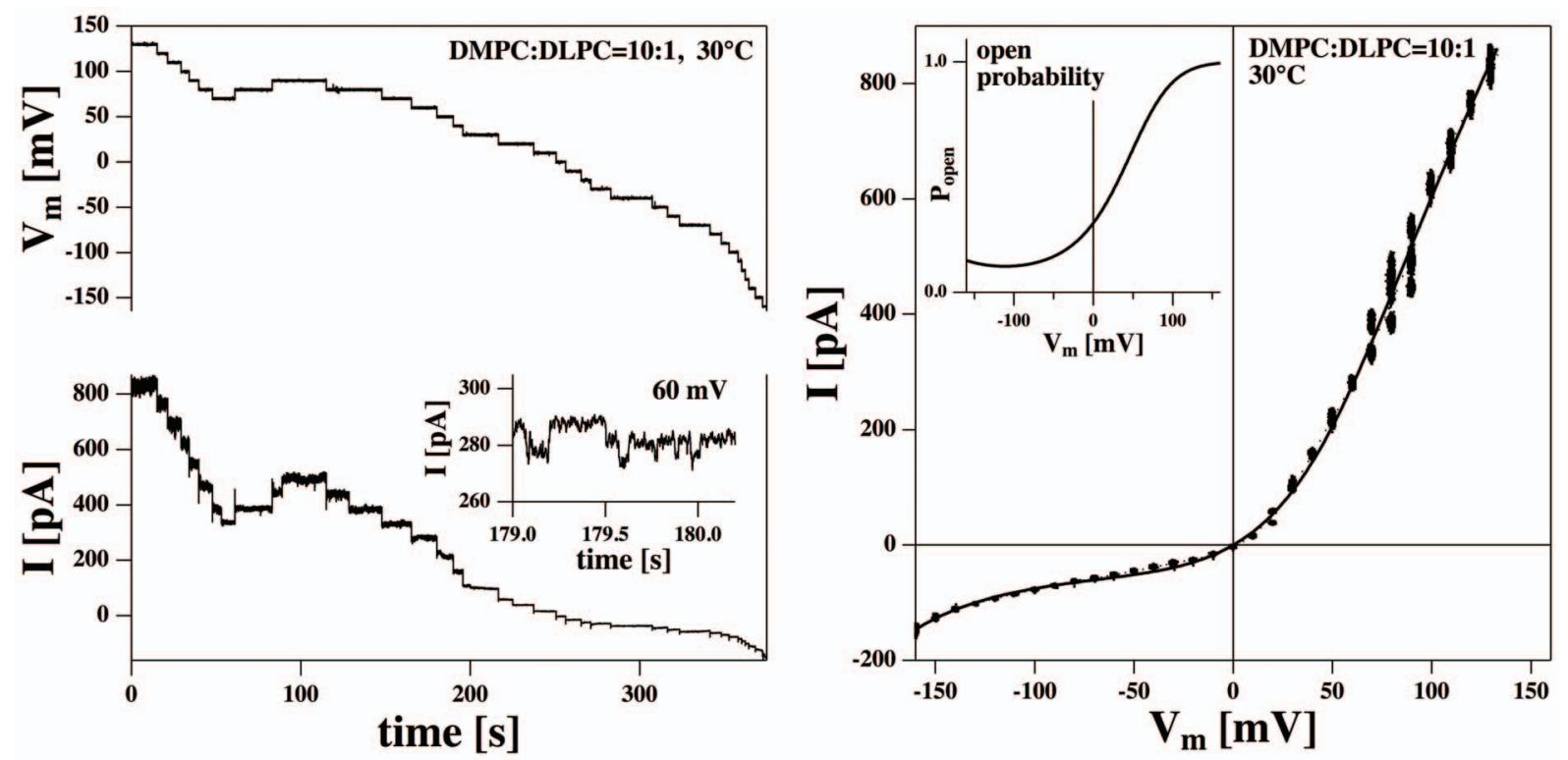

Figure 1. Current-voltage profile of a DMPC:DLPC $=10: 1 \mathrm{~mol}: \mathrm{mol}$ membrane at $30^{\circ} \mathrm{C}$. Left: Membrane voltage and the corresponding total membrane current as a function of time. The voltage was changed stepwise during the experiment. The insert shows some small channel events at $\mathrm{V}_{m}=60 \mathrm{mV}$ with a conductance of about $\gamma=125 \mathrm{pS}$. Right: Resulting current-voltage relation. The I-V curve is outward rectified. The solid line is a fit to eq. 6 (parameters are given in the text). The insert shows the corresponding open probability of a membrane pore deduced from eq. 5. It displays a minimum at $V_{0}=-110 \mathrm{mV}$.

doi:10.1371/journal.pone.0065707.g001

gating of the lipid channels is a generic phenomenon whenever lipid channels can be recorded, it is rather rare that a complete I$\mathrm{V}$ curve can be investigated over a long period without drifts or breaking of the membrane.

Fig. 2 shows another experiment performed at the same conditions as the one in Fig. 2. Panel A contains a section of about 25 minutes of the time dependence of both voltage and membrane current (the total experiment lasted more than an hour). Panels $\mathrm{B}_{1}$ and $\mathrm{B}_{2}$ (containing a magnification of $\mathrm{B}_{1}$ ) show the $\mathrm{I}-\mathrm{V}$ plot using the total raw data $\left(\mathrm{V}_{m}\right.$ ranging from $-250 \mathrm{mV}$ to $\left.+100 \mathrm{mV}\right)$. At positive voltages, one can recognize individual peaks in the distribution, corresponding to at least five individual current steps. Four events at $\mathrm{V}_{m}=50 \mathrm{mV}$ are highlighted in the red box in panels $\mathrm{A}$ and $\mathrm{B}_{2}$. The corresponding amplified current trace is shown in panel $\mathrm{C}_{1}$ and consecutive enlargements are shown in panels $\mathrm{C}_{2}$ to $\mathrm{C}_{4}$. One can clearly recognize four individual current levels, which must be attributed to at least 2-3 different pores with open and closed states. The difference in current between these four steps corresponds to three conductances of $\gamma \sim 240 \mathrm{pS}$, $\gamma \sim 900 \mathrm{pS}$ and $\gamma \sim 320 \mathrm{pS}$ (extracted from panel C2).

The data in Fig. 3 (bottom) represent the two current steps with the largest conductance shown in Fig. 2 (cf, panel $\mathrm{C}_{4}$ ), recorded at four different voltages. The recordings were quite stable showing permanent opening and closing of channels over up to 30 minutes for a single voltage. Increasing the voltage leads to a larger singlechannel current and to an increased likelihood of finding open channels. The current-voltage relation for the single-channel current is linear (Fig. 3, top), indicating the presence of lipid channels with a constant pore size. The single-channel conductance is $\gamma=320 \mathrm{pS}$ and does not seem to be voltage-dependent. We could not resolve any single-channel activity at negative voltages indicating an asymmetric membrane. The traces allow for an accurate statistical analysis of the single channel events. Fig. 4 (top) shows the current histograms associated with the current traces of Fig. 3. One finds two peaks in the distribution representing the closed and the open states. The current of the closed state was set to zero. The (voltage-dependent) equilibrium constant, $K\left(V_{m}\right)$, between open and closed state can be deduced from the peak areas of the two states in the histogram. From the equilibrium constant one can deduce the free energy difference of the two states (Fig. 4, bottom left) and the open probability (Fig. 4 bottom right) - see eq. 5. The solid lines in the bottom panels represent fits assuming a quadratic form of the free energy as given by eq. 4 with an offset voltage of $V_{0}=-110 \pm 60 \mathrm{mV}$, $\Delta G_{0}=21.3 \mathrm{~kJ} / \mathrm{mol}$ and $\alpha=-721 \mathrm{~kJ} / \mathrm{mol} \cdot \mathrm{V}^{2} . \mathrm{V}_{0}$ is consistent with the offset voltage obtained from the $\mathrm{I}-\mathrm{V}$ profile in Fig. 1 . It is also in agreement with the observation that one does not find any channel events at negative voltages in the regime investigated here. $\Delta G_{0}$ and $\alpha$ assume larger values as for the $\mathrm{I}-\mathrm{V}$ profile in Fig. 1 which indicates that the pores are larger. Fig. 4 indicates that one finds a voltage-induced transition from a closed to an open state over a range of about $30 \mathrm{mV}$. Such a range is also typical for the activation protein channels. For instance, the voltage-gated $\mathrm{K}^{+}$channel HERG [46] displays an closed-open transition with a similar width [46]. If one attributes the open-closed event to a single protein, one typically would associate it to a gating charge moving in a field. The open-probability shown in Fig. 4 (bottom right) can be reasonably well described by such a model. The equilibrium between a closed and an open channel described by a gating charge $z$ is given by $K=\exp \left[-\left(\Delta G_{0}+z F V_{m}\right) / R T\right]$. The best fit yields $z \approx 2.5$, which is of the same order as values found for many proteins. However, as we have shown in [34] and in the theory section, the gating could also originate from charging the membrane capacitor as a whole.

\section{Traces with two steps}

In Fig. 5 the voltage and current traces of a different membrane (DMPC:DLPC $=10: 1 ; \mathrm{T}=29^{\circ} \mathrm{C}$ ) are displayed together with the corresponding $\mathrm{I}-\mathrm{V}$ plot (right panel), recorded at positive voltages only. In this plot, three current levels can nicely be resolved. At 

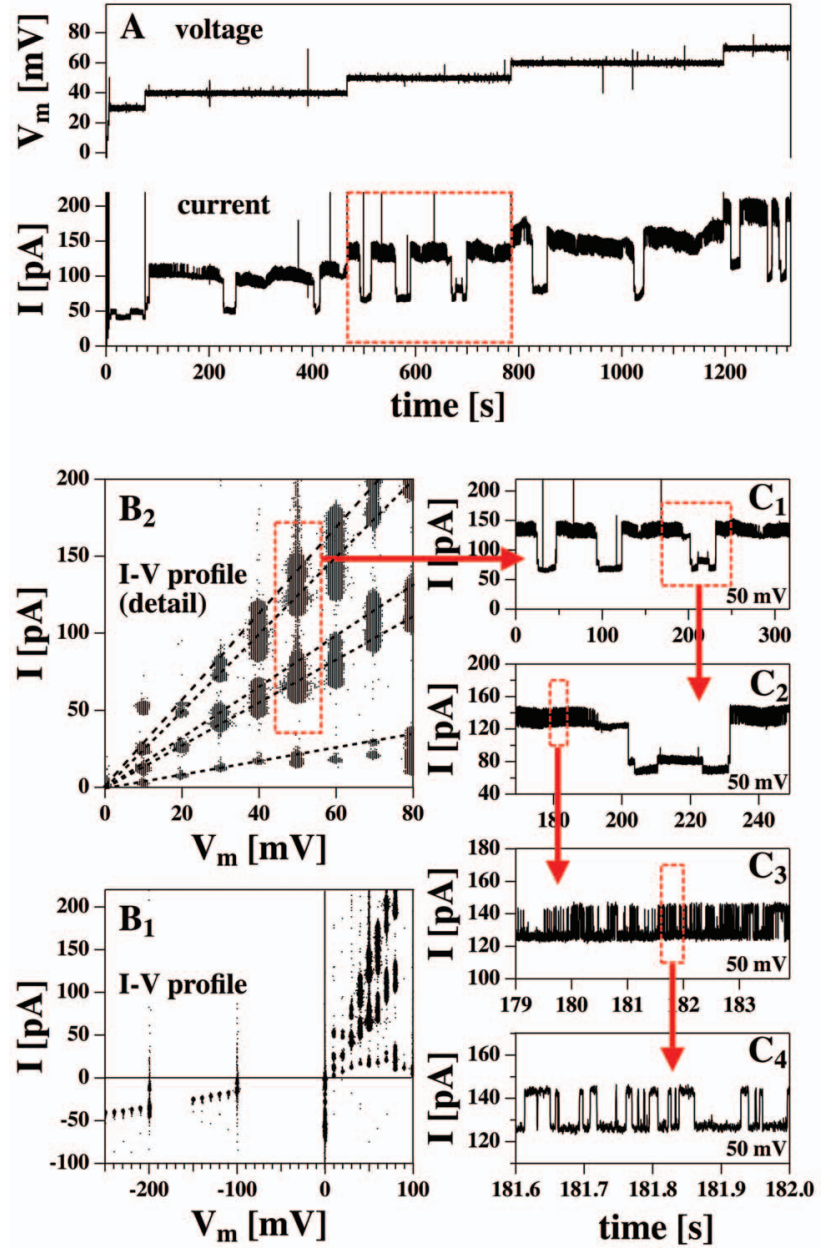

Figure 2. Current-voltage behavior of a different preparation of a DMPC:DLPC $=10: 1 \mathrm{~mol}: \mathrm{mol}$ membrane at $30^{\circ} \mathrm{C}$ showing many channel events. A: Section of the raw voltage and current traces (20 minutes out of 1 hour recording). The section in the red box is amplified in panel $C_{1}$. $B_{1}: I-V$ profile of the complete data set. At positive voltages one can recognize $\sim 5$ individual current levels. $B_{2}$ : Amplified section of panel $B_{1}$. The black dashed lines are guides to the eye to mark the five current levels. The red box marks the four current levels that can be seen in panel $C_{1}$. $C_{1}$ : Current I at $V_{m}=50 \mathrm{mV}$ over 5 minutes. Panels $C_{2}-C_{4}$ : Consecutive enlargement of small sections of the profile in panel $C_{1}$. In panel $C_{4}$, two individual currents levels can easily be recognized. These two levels are investigated in more detail in Fig. 3.

doi:10.1371/journal.pone.0065707.g002

some voltages (30 and $40 \mathrm{mV}$ ) these pores coexist. As in the previous examples, the current at each of these levels displays a linear current-voltage relation (indicated by the straight dashed lines in Fig. 5, right). However, the likelihood to find any one of these levels is voltage-dependent.

Fig. 6 (left) shows details of the current trace in Fig. 5 at 6 different voltages from 30 to $55 \mathrm{mV}$. In the $40 \mathrm{mV}$ trace one can recognize two steps of different magnitude. At lower voltages, a large pore with a single-channel conductance of $\gamma \sim 831 \mathrm{pS}$ is found, whereas at high voltages one finds a different pore with $\gamma \sim 287$ pS. Overall, the high voltage channel has a smaller singlechannel conductance as the low voltage channel. As in Fig. 2 the large pore displays longer open and closed times. The right hand panel shows the histograms of the first and the second voltage step as a function of voltage. Zero pA corresponds to the baseline
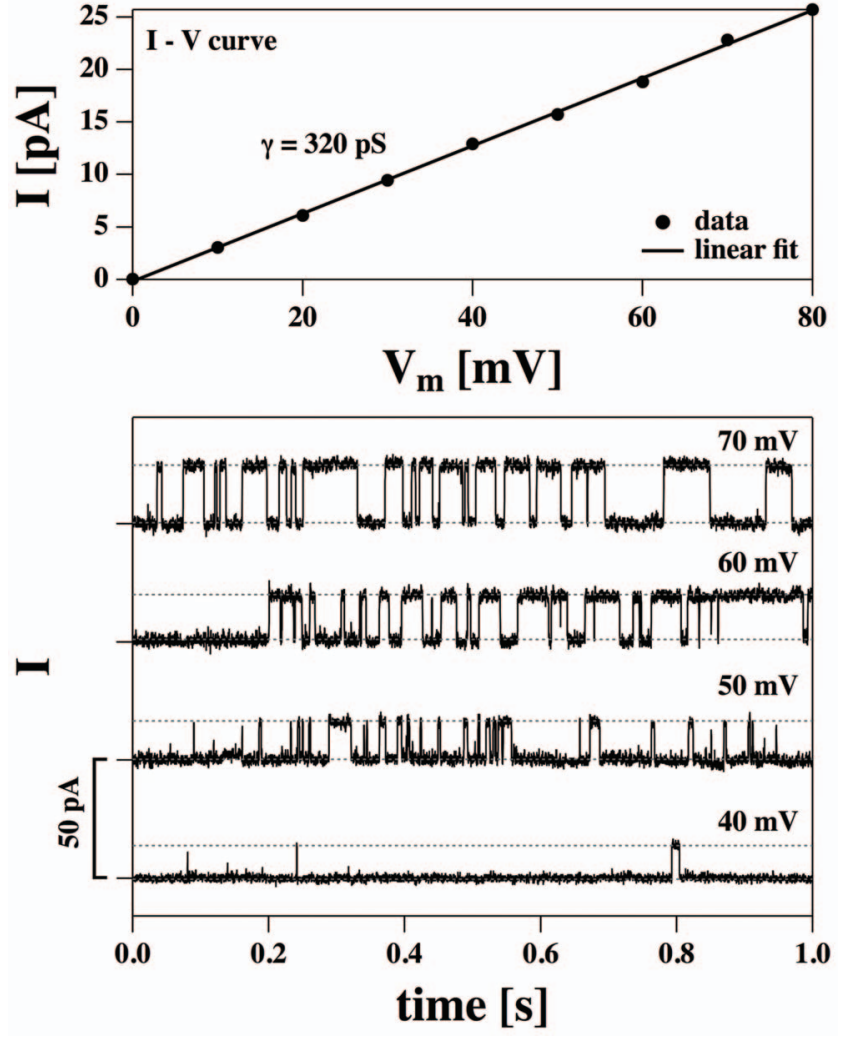

Figure 3. Recordings of the DMPC:DLPC $=10: 1$ membrane from Fig. 2 as a function of voltage (cf. Fig. 2, panel C4). Bottom: Current-traces for a DMPC:DLPC $=10: 1 \mathrm{~mol} / \mathrm{mol}$ membrane $(150 \mathrm{mM}$ $\mathrm{KCl}, \mathrm{T}=30^{\circ} \mathrm{C}$ ) at four voltages showing an increase of single-channel conductance with voltage and an increased likelihood of channel formation. Top: The corresponding linear single-channel currentvoltage relation indicating a single-channel conductance of $\gamma=320 \mathrm{pS}$. doi:10.1371/journal.pone.0065707.g003

current. The closed-to-open transition of the first step takes place at $\sim 34 \mathrm{mV}$. The transition of the second step is found at $\sim 50 \mathrm{mV}$. Here, zero pA corresponds to the current of the first open channel. The free energy of the two steps as a function of voltage is given in Fig. 6 (bottom right). The free energy of step 2 represents that of the single channel (i.e., it is the difference in free energy between the first and second open event). Both curves are well approximated by quadratic functions with $\mathrm{V}_{0}=0 \mathrm{mV}$. At zero voltage, the free energy of the second pore $\left(\Delta G_{0,2}\right)$ has been found to be approximately twice the free energy of one pore $\left(\Delta G_{0,1}=25.4 \mathrm{~kJ} / \mathrm{mol} ; \alpha_{1}=-22.2 \cdot 10^{3} \mathrm{~kJ} /\right.$ $\left.\mathrm{mol} \cdot \mathrm{V}^{2} ; \Delta G_{0,2}=46.9 \mathrm{~kJ} / \mathrm{mol} ; \alpha_{2}=-18.6 \cdot 10^{3} \mathrm{~kJ} / \mathrm{mol} \cdot \mathrm{V}^{2}\right)$. This is also reflected in the fact that the second pore forms at a higher voltage. The parameter $\alpha$ describing the voltage dependence of each pore is the same within error.

As mentioned already in the Materials section, our patch pipette method yields qualitative similar results in recordings for different membranes or methods. We found similar channel events in BLM recordings, when using patch pipettes with sharp edges, and with fire-polished pipettes. This indicates that the appearance of the channels as such is not primarily due to the nature of a particular interface. However, it is possible that channel probabilities are altered by the presence of pipette or Teflon interfaces or by other membrane perturbations, and we argued earlier that the domain interfaces and proteins could also represent such perturbations [1]. All of these perturbations may locally alter membrane fluctuations. The experiments display significant quantitative variations between preparations. This implies, that lipid channels may display 

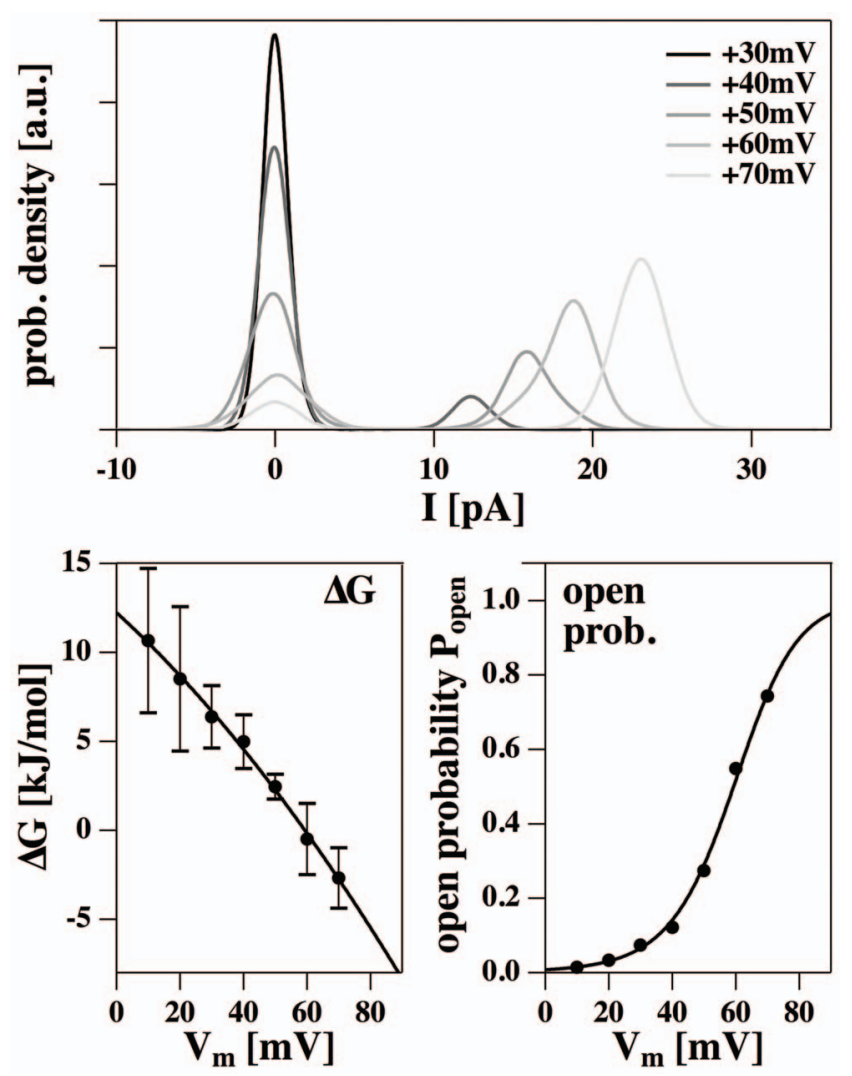

Figure 4. Statistical analysis of the data in Fig. 4. Top: Experimental probability distribution for channel opening at five voltages (cf. Fig. 4). Bottom, left: The calculated free energy difference between open and closed state as a function of voltage indicates a quadratic voltage dependence (see eq. $4, \Delta G_{0}=21.3 \mathrm{~kJ} / \mathrm{mol}$; $\alpha=-721 \mathrm{~kJ} / \mathrm{mol} \cdot \mathrm{V}^{2}, \mathrm{~V}_{0}=-110 \mathrm{mV}$ ). Bottom, right: The open probability, $P_{\text {open }}$, shows a transition at $\sim 60 \mathrm{mV}$.

doi:10.1371/journal.pone.0065707.g004 somewhat different conductances and voltage-dependences from experiment to experiment. The reason for this is not exactly clear. We have speculated that under the conditions used by us the lipid mixtures may display domains that are of similar size than the patch diameter. Domain sizes that are visible under a microscope are quite common in binary lipid mixtures close to melting transitions. This may lead to variations of the fluid membrane fraction in different experiment. As discussed above, another possibility is that the patch pipette itself has an influence on the physical behavior of the membranes as is known for the interaction of membranes with glass surfaces [47]. A third possibility is that the membranes in the patch pipette are curved due to differences of pressure on both sides of the membrane. Petrov and collaborators have argued that membrane curvature is coupled to the generation of a transmembrane voltage $V_{0}$ due to a phenomenon called 'flexoelectricity' [48]. In the case of a spherically curved bilayer with radius of curvature $c$, Petrov finds that

$$
V_{0}=\frac{2}{\epsilon_{0}} f c
$$

where $\epsilon_{0}=8.854 \cdot 10^{-12} \mathrm{~F} / \mathrm{m}$ is the vacuum permittivity and $f$ is a parameter determined from experiment [48]. The value found by Petrov for egg yolk phosphatidylcholine in $0.1 \mathrm{M} \mathrm{KCl}$ was $f=13 \cdot 10^{-19} \mathrm{C}$ (Coulombs). Consequently, an offset potential of the order of $V_{0}=100 \mathrm{mV}$ (as found in our present experiments) would be generated by a small radius of curvature of about $R=1 / c=3 \mu m$. Such a curvature seems plausible for a membrane patch with a diameter of $\sim 1 \mu \mathrm{m}$ in the presence of slight suction. A BLM with a diameter of $80 \mu \mathrm{m}$ possesses a minimum radius of $R=40 \mu \mathrm{m}$, which corresponds to a maximum voltage caused by flexoelectricity of $V_{0}=7 \mathrm{mV}$. It is therefore expected that the offset voltage created by flexoelectricity is small for BLMs but putatively large for small patch pipettes as found by [29]. If pipette suction is not controlled, the channel open-probabilities would also
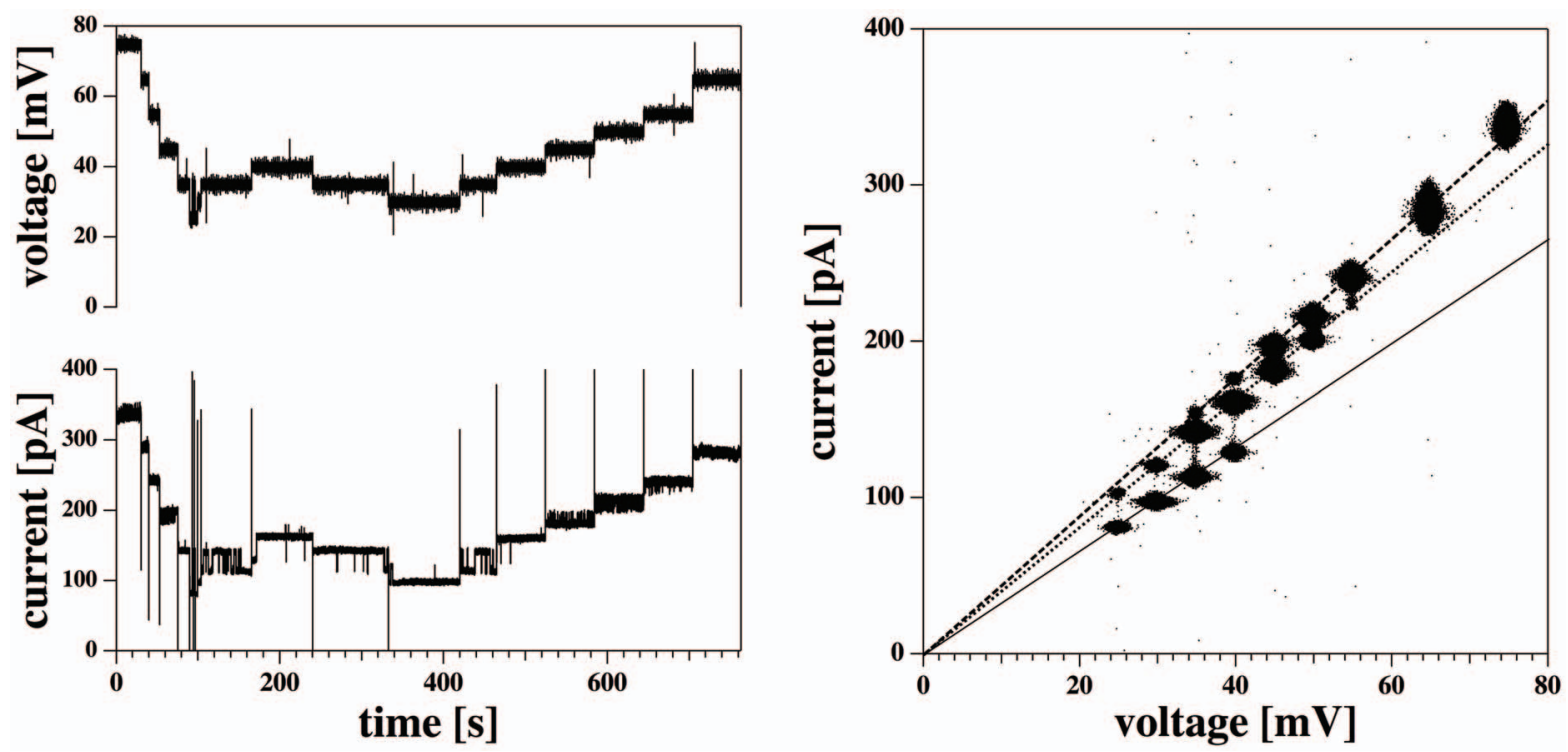

Figure 5. Recordings on a DMPC:DLPC membrane at $29^{\circ} \mathrm{C}$. Top: Probability distribution for the first (left) and the second open state (right). Bottom: The calculated free energy difference for both conduction steps is consistent with similar quadratic voltage dependence (for parameters see text).

doi:10.1371/journal.pone.0065707.g005 

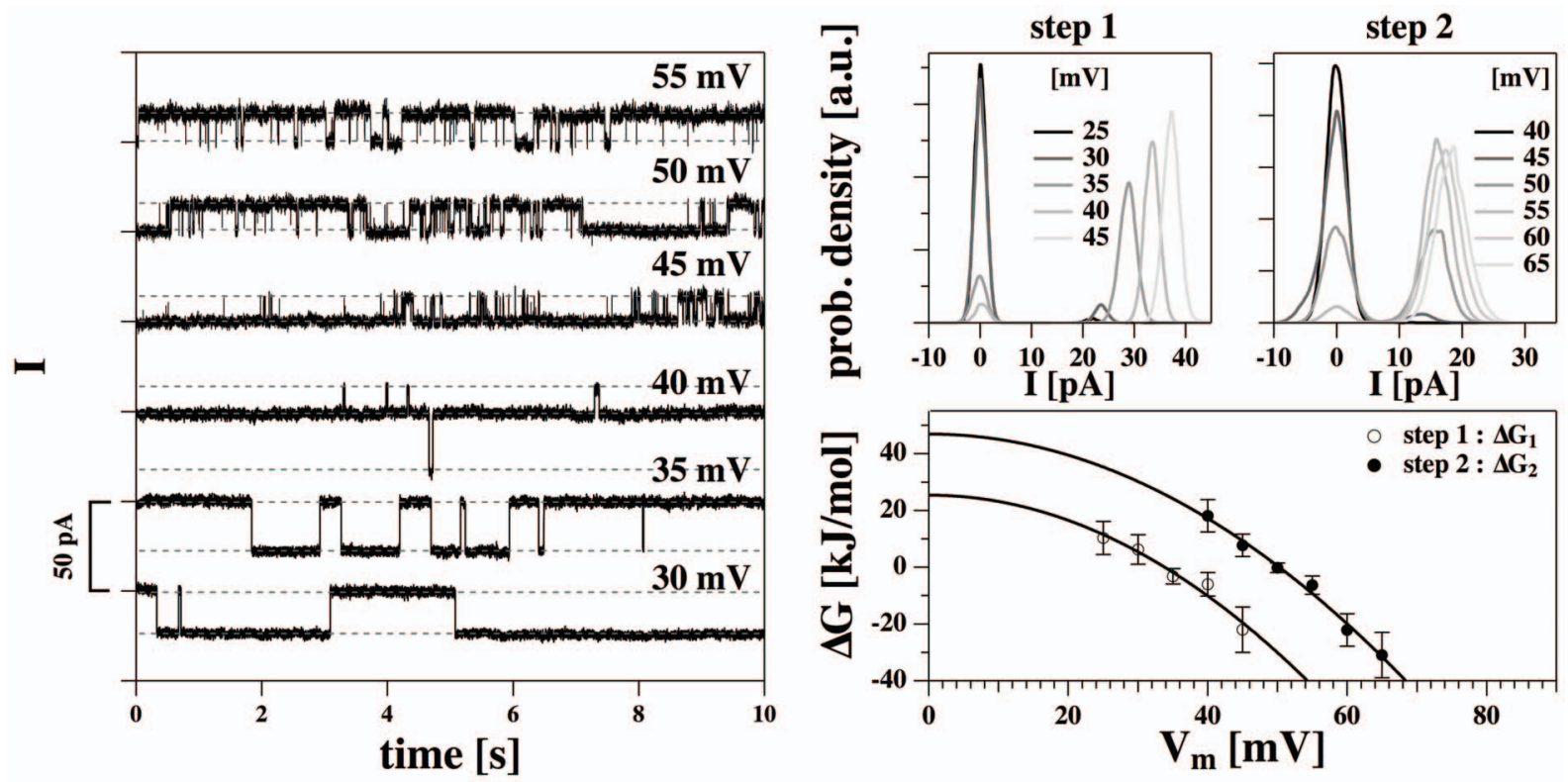

Figure 6. Recordings on a DMPC:DLPC membrane at $29^{\circ} \mathbf{C}$. Left: Current traces at 6 voltages from $30 \mathrm{mV}$ to $55 \mathrm{mV}$. THe trace at $40 \mathrm{mV}$ shows twos large and a small current step. Right, top: Probability distribution for the first (left) step with a conductance of $\gamma=830 \mathrm{pS}$ and the second open state (right) with a conductance of $\gamma=287 \mathrm{pS}$. Bottom: The calculated free energy difference for both conduction steps is consistent with a nearly identical quadratic voltage dependence $\left(\Delta G_{0,1}=25.4 \mathrm{~kJ} / \mathrm{mol} ; \alpha_{1}=-22.2 \cdot 10^{3} \mathrm{~kJ} / \mathrm{mol} \cdot \mathrm{V}^{2} ; \Delta G_{0,2}=46.9 \mathrm{~kJ} / \mathrm{mol} ; \alpha_{2}=-18.6 \cdot 10^{3} \mathrm{~kJ} / \mathrm{mol} \cdot V^{2}\right)$. doi:10.1371/journal.pone.0065707.g006

not be well-defined, which may well explain the variations in our experiments.

It should be added the all of the above sources for the facilitation of ion channels are also present in patch-clamp experiments on biological preparations. For the above reasons, however, absolute numbers and the differences in conductances and free energies of pore formation for different membranes (as in Figs. 4 and 6) should not be over-interpreted. Within one preparation, however, all results are quantitatively reproducible. They all display voltage gating with a quadratic voltagedependence of the open probability while simultaneously the single-channels conductances remain constant.

\section{Lifetime distributions}

The quite long channel traces shown in Figs. 2 and 5 allow for a detailed lifetime analysis of the lipid channels. In Fig. 7 the probability distribution functions (PDF) of channel open- and closed-times is given for the channels shown in Fig. 3 (DMPC:DLPC $=10: 1 ; \mathrm{T}=30^{\circ} \mathrm{C}$ ) at four different voltages. Panels $A$ and $B$ show a linear representation of the distributions for the open and closed lifetimes in the interval up to $100 \mathrm{~ms}$. In the interval up to $100 \mathrm{~ms}$ these curves can seemingly be reasonably well described by a double-exponential function. For instance, a fit of the closed distribution at $60 \mathrm{mV}$ yields two lifetimes, 2.6 and $13.7 \mathrm{~ms}$, respectively. However, closer inspection of the PDFs in a $\log$-log representation (panels $C$ and $D$ ) shows that the distributions range over several orders of magnitude and clearly display nonexponential behavior. Single-exponential behavior would be expected for statistical independent Markov-processes of two-state state conversions. However, at long open times, the slope in the open-time PDF approaches a $t^{-3 / 2}$ power law behavior, which we found earlier in other synthetic lipid membrane preparations [24]. A possible theoretical scheme for the open time PDF leading to such a power law given is

$$
C \stackrel{k}{\leftarrow} O_{1} \stackrel{K}{\rightleftharpoons} O_{2} \stackrel{K}{\rightleftharpoons} O_{3} \stackrel{K}{\rightleftharpoons} \quad \ldots \quad \stackrel{K}{\rightleftharpoons} O_{N}
$$

where $C$ is a closed state and $O_{1}, O_{2}, \ldots O_{N}$ are $\mathrm{N}$ open states of a lipid pore with equal conductance, and the equilibrium constants between open states $K=O_{i+1} / O_{i}$ are constant. A similar fractal behavior was found also for proteins - for instance for voltagegated potassium channels [49]. Originally, the above scheme was designed to describe such protein channels [50] by assuming $N$ open states of a channel protein. This means that in respect to open-time distributions, lipid and protein channels are quite similar. Our results show that fitting the open-time PDF with a biexponential function as commonly done for protein channels may be quite misleading. The PDFs show that such a description would largely underestimate the occurrence of very long open and closed events, respectively.

A fractal nature of the PDFs must not necessarily originate from a discrete scheme as shown in eq. 9. All of our experiments take place close to melting transitions, and it has be shown that the transition itself display fractal characteristics in terms of cooperative spacial density fluctuations [51] and most likely of temporal patterns, too.

The distribution profiles recorded at the four different voltages are seemingly very similar in the linear representation at short times. However, in the log-log representation it becomes obvious that especially the long open-times are significantly more frequent at higher voltage, while simultaneously the long closed events become less frequent. There is a finite probability to find very long open- or closed-times. The latter may give rise to silent periods in the conductance recordings followed by activity burst that have also been found in both in synthetic lipid membranes and protein channel-containing preparations [28]. 

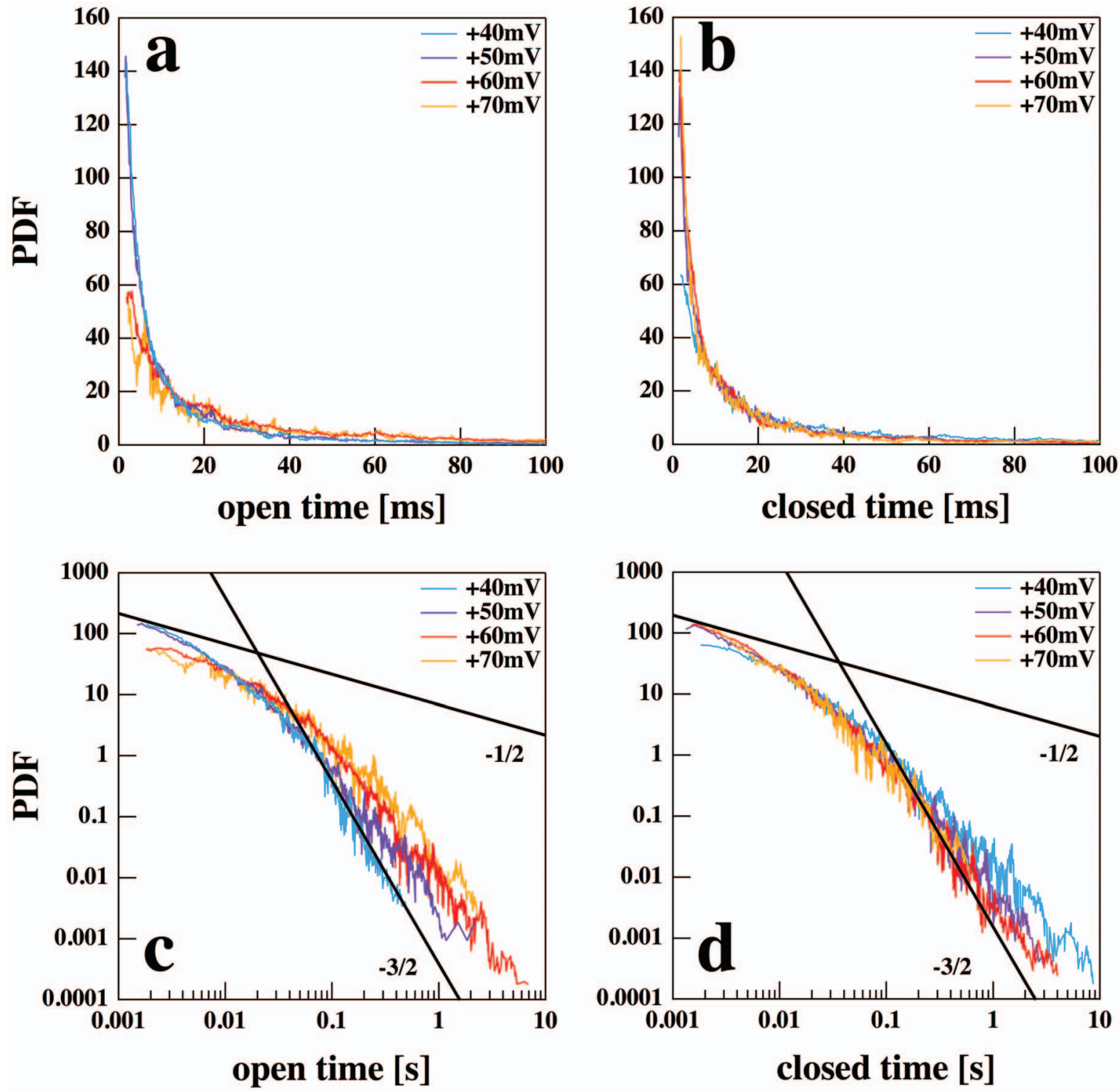

Figure 7. Open and closed lifetimes of the lipid ion channels for the traces at four different voltages shown in Fig. 4. (A) Probability distribution functions (PDF) of the channel open-times at short times of up to $100 \mathrm{~ms}$. (B) Closed time PDF. (C) and (D). Double-logarithmic representation of panels $A$ and $B$ over time scales up to 10 seconds. One can recognize that the PDFs are non-exponential, but also do not obey a simple power law in the double-log plot. The solid lines are guides to the eye with slopes of $-1 / 2$ and $-3 / 2$. At long times the pdf seem to approach a $t^{-3 / 2}$ behavior.

doi:10.1371/journal.pone.0065707.g007

\section{Conclusions}

We have shown that lipid ion channels in model membranes are voltage-gated. This is in agreement with previous reports on the effect of electrostriction on membrane thinning [30,34,40]. The free energy of the open pore has a quadratic dependence on voltage. Such a quadratic voltage dependence is characteristic for the charging of a capacitor with electrical breakthroughs at critical voltages. It differs from a standard Eyring transition state model that considers movement of charges in a field. We could excellently describe the $\mathrm{I}-\mathrm{V}$ profiles of membranes with our simple channel activation model when assuming an asymmetric membrane. The conductances of single channel events $(\gamma=125-$ $900 \mathrm{pS}$ in the present experiments) and the closed-to-open transition width are of a similar order than the results found for protein channels. The size of the lipid pores does not seem to be fixed but varies between preparations. However, it is constant during one experiment. Our results complement earlier findings that lipid channels can be gated by other intensive variables, e.g., anesthetic drugs, temperature, $\mathrm{pH}$, and calcium [1]. The dependence of channel formation on these variables strongly suggests that these events are related to the well-understood physical chemistry of lipid membranes. Increasing voltage favors channel openings with long time scales.

The quantitative similarities between lipid and protein channels are intriguing. While they could be absolutely fortuitous, we find it more likely that these similarities are not accidental but rather suggest that conduction events have a common origin in the thermodynamics of the biomembrane. 


\section{Acknowledgments}

Andrew D. Jackson from the Niels Bohr International Academy kindly proof-read our manuscript. We thank one of the reviewers for pointing out the possibility of an asymmetric membrane. Karis Zecchi from the Membrane Biophysics Group provided the value for the offset voltage expected from flexoelectricity.

\section{References}

1. Heimburg T (2010) Lipid ion channels. Biophys Chem 150: 2-22.

2. Yafuso M, Kennedy SJ, Freeman AR (1974) Spontaneous conductance changes, multilevel conductance states and negative differential resistance in oxidized cholesterol black lipid membranes. J Membr Biol 17: 201-212.

3. Antonov VF, Petrov VV, Molnar AA, Predvoditelev DA, Ivanov AS (1980) The appearance of single-ion channels in unmodified lipid bilayer membranes at the phase transition temperature. Nature 283: 585-586.

4. Kaufmann K, Silman I (1983) Proton-induced ion channels through lipid bilayer membranes. Naturwissenschaften 70: 147-149.

5. Kaufmann K, Silman I (1983) The induction by protons of ion channels through lipid bilayer membranes. Biophys Chem 18: 89-99.

6. Göogelein H, Koepsell H (1984) Channels in planar bilayers made from commercially available lipids. Pügers Arch 401: 433-434.

7. Antonov VF, Shevchenko EV, Kozhomkulov ET, Molnar AA, Smirnova EY (1985) Capacitive and ionic currents in BLM from phosphatidic acid in $\mathrm{Ca}^{2+}$ induced phase transition. Biochem Biophys Research Comm 133: 1098-1103.

8. Kaufmann K (1989) Lipid membrane. http://membranes.nbi.dk/Kaufmann/ pdf/-Kaufmann book5 org.pdf, Caruaru.

9. Antonov VF, Smirnova EY, Shevchenko EV (1990) Electric field increases the phase transition temperature in the bilayer membrane of phosphatidic acid. Chem Phys Lipids 52: 251-257.

10. Antonov VF, Anosov AA, Norik VP, Smirnova EY (2005) Soft perforation of planar bilayer lipid membranes of dipalmitoylphosphatidylcholine at the temperature of the phase transition from the liquid crystalline to gel state. Eur Biophys J 34: 155-162.

11. Siskind LJ, Colombini M (2000) The lipids $\mathrm{c}_{2}$ - and $\mathrm{c}_{16}$-ceramide form large stable channels. Implications for apoptosis. J Biol Chem 275: 38640-38644.

12. Siskind LJ, Kolesnick RN, Colombini M (2002) Ceramide channels increase the permeability of the mitochondrial outer membrane to small proteins. J Biol Chem 277: 26796-26803.

13. Siskind LJ, Davoody A, Lewin N, Marshall S, Colombini M (2003) Enlargement and contracture of $\mathrm{c}_{2}$-ceramide channels. Biophys J 85: 1560-1575.

14. Siskind LJ, Kolesnick RN, Colombini M (2006) Ceramide forms channels in mitochondrial outer membranes at physiologically relevant concentrations. Mitochondrion 6: 118-125.

15. ColombiniM(2010) Ceramide channels and their role in mitochondria-mediated apoptosis. Biochim Biophys Acta 1797: 1239-1244.

16. Perera MN, Ganesan V, Siskind LJ, Szulc ZM, Bielawski J, et al. (2012) Ceramide channels: Inuence of molecular structure on channel formation in membranes. Biochim Biophys Acta 1818: 1-11.

17. Shao C, Sun B, DeVoe DL, Colombini M (2012) Dynamics of ceramide channels detected using a microuidic system. PloS ONE 7: e43513.

18. Heimburg $T$ (1998) Mechanical aspects of membrane thermodynamics. Estimation of the mechanical properties of lipid membranes close to the chain melting transition from calorimetry. Biochim Biophys Acta 1415: 147-162.

19. Ebel H, Grabitz P, Heimburg T (2001) Enthalpy and volume changes in lipid membranes. i. the proportionality of heat and volume changes in the lipid melting transition and its implication for the elastic constants. J Phys Chem B 105: 7353-7360.

20. Grabitz P, Ivanova VP, Heimburg T (2002) Relaxation kinetics of lipid membranes and its relation to the heat capacity. Biophys J 82: 299-309.

21. Seeger HM, Gudmundsson ML, Heimburg T (2007) How anesthetics, neurotransmitters, and antibiotics inuence the relaxation processes in lipid membranes. J Phys Chem B 111: 13858-13866.

22. Blicher A, Wodzinska K, Fidorra M, Winterhalter M, Heimburg T (2009) The temperature dependence of lipid membrane permeability, its quantized nature, and the inuence of anesthetics. Biophys J 96: 4581-4591.

23. Wunderlich B, Leirer C, Idzko A, Keyser UF, Myles V, et al. (2009) Phase state dependent current uctuations in pure lipid membranes. Biophys J 96: 45924597.

24. Gallaher J, Wodzinska K, Heimburg T, Bier M (2010) Ion-channel-like behavior in lipid bilayer membranes at the melting transition. Phys Rev E 81: 061925.

\section{Author Contributions}

Conceived and designed the experiments: AB TH. Performed the experiments: AB. Analyzed the data: $\mathrm{AB}$ TH. Wrote the paper: $\mathrm{TH}$.

25. Heimburg T, Jackson AD (2005) On soliton propagation in biomembranes and nerves. Proc Natl Acad Sci USA 102: 9790-9795.

26. Heimburg T (2007) Thermal biophysics of membranes. Berlin, Germany: Wiley VCH.

27. Heimburg T, Jackson AD (2007) The thermodynamics of general anesthesia. Biophys J 92: 3159-3165.

28. Laub KR, Witschas K, Blicher A, Madsen SB, Lückhoff A, et al. (2012) Comparing ion conductance recordings of synthetic lipid bilayers with cell membranes containing trp channels. Biochim Biophys Acta 1818: 1-12.

29. Wodzinska K, Blicher A, Heimburg T (2009) The thermodynamics of lipid ion channel formation in the absence and presence of anesthetics. blm experiments and simulations. Soft Matter 5: 3319-3330.

30. Crowley JM (1973) Electrical breakdown of bimolecular lipid membranes as an electromechanical instability. Biophys J 13: 711-724.

31. Abidor IG, Arakelyan VB, Chernomordik LV, Chizmadzhev YA, Pastushenko VF, et al. (1979) Electric breakdown of bilayer lipid-membranes. 1. Main experimental facts and their qualitative discussion. Bioelectrochem Bioenerg 6: 37-52.

32. Winterhalter M, Helfrich W (1987) Effect of voltage on pores in membranes. Phys Rev A 36: 5874-5876.

33. Neumann E, Kakorin S, Tænsing K (1999) Fundamentals of electroporative delivery of drugs andgenes. Bioelectrochem Bioenerg 48: 3-16.

34. Heimburg T (2012) The capacitance and electromechanical coupling of lipid membranes close to transitions. the effect of electrostriction. Biophys J 103: 918929.

35. Hanke W, Methfessel C, Wilmsen U, Boheim G (1984) Ion channel reconstruction into lipid bilayer membranes on glass patch pipettes. Bioelectrochem Bioenerg 12: 329-339.

36. Blicher A (2011) Electrical aspects of lipid membranes. Ph.D. thesis, University of Copenhagen. http://www.nbi.ku.dk/english/research/phd-theses/phdtheses-2011/andreas-blicher/andreas_blicher.pdf. Accessed 1013 May 06.

37. Sigworth FJ, Sine SM (1987) Data transformations for improved display and fitting of singlechannel dwell time histograms. Biophys J 52: 1047-1054.

38. Stark JA, Hladky SB (2000) Adjustments for the display of quantized ion channel dwell times in histograms with logarithmic bins. Biophys J 78: 662-667.

39. Liebovitch L, Scheurle D, Rusek M, Zochowski M (2001) Fractal methods to analyze ion channel kinetics. Methods 24: 359-375.

40. White SH, Thompson TE (1973) Capacitance, area, and thickness variations in thin lipid films. Biochim Biophys Acta 323: 7-22.

41. Glaser RW, Leikin SL, Chernomordik LV, Pastushenko VF, Sokirko AI (1988) Reversible breakdown of lipid bilayers: Formation and evolution of pores. Biochim Biophys Acta 940: 275-287.

42. Alvarez O, Latorre R (1978) Voltage-dependent capacitance in lipid bilavers made from monolayers. Biophys J 21: 1-17.

43. Johnston D, Wu SMS (1995) Cellular Neurophysiology. Boston: MIT Press.

44. DMPC - dimyristoyl phophatidylcholine; DLPC - dilauroyl phosphatidylcholine.

45. Mosgaard LD, Heimburg T (2013) Lipid ion channels and the role of proteins. Submitted.

46. Trudeau MC, Warmke JW, Ganetzky B, Robertson CA (1995) HERG, a human inward rectifier in the voltage-gated potassium channel family. Science 269: 92-95.

47. Yang J, Appleyard J (2000) The main phase transition of mica-supported phosphatidylcholine membranes. J Phys Chem B 104: 8097-8100.

48. Petrov AG (2001) Flexoelectricity of model and living membranes. Biochim Biophys Acta 1561: 1-25.

49. Liebovitch LS, Sullivan JM (1987) Fractal analysis of a voltage-dependent potassium channel from cultured mouse hippocampal neurons. Biophys J 52: 979-988.

50. Millhauser GL, Salpeter EE, Oswald RE (1988) Diffusion models of ion-channel gating and the origin of power-law distributions from single-channel recording. Proc Natl Acad Sci USA 85: 1503-1507.

51. Nielsen LK, Bjørnholm T, Mouritsen OG (2000) Critical phenomena uctuations caught in the act. Nature 404: 352. 